\title{
The Establishment of base Model of UAV Propeller
}

\author{
Fupei Zheng ${ }^{1, a^{*}}$, Song $\mathrm{Li}^{2, \mathrm{~b}}$ \\ ${ }^{1}$ Air Force Xi'an Flight Academy, Xian, China \\ ${ }^{2}$ Air Force Aviation University, Jilin, China \\ az-f-p@126.com, blisong4885@126.com \\ *Corresponding author
}

Keywords: UAV, propeller, modeling, Propeller

\begin{abstract}
UAV propeller geometry type surface is more complex, in this paper, on the basis of previous preliminary design of the UAV propeller based model calculation results, the use of computer information technology means of propeller geometry model was built first, which contains some practical experience and skills, for subsequent research on modeling and aerodynamic performance of UAV propeller calculation can provide certain reference.
\end{abstract}

\section{Introduction}

Unmanned aerial vehicle (UAV) has become a leading edge and hotspot in the research of the aviation industry at present, its cost is low, can carry a certain aviation equipment, buffet for disaster relief, field investigation, monitoring and prevention and cure of diseases and pests of agriculture and forestry, film and television entertainment, scientific research and other fields have broad application prospects. The drones seen now rely on propellers as thrusters to maneuver, either in the wings or in the gyrocopters. Because the propeller aerodynamic profile is irregular, geometric model is established at the beginning will often meet some difficulties, this paper mainly illustrates how the propeller model is built at the beginning of modeling for UAV propulsion research support, this model can also according to the calculation of expansion.

\section{Establishment of Airfoil}

This part is the propeller of the three dimensional geometry data model on the basis of establishing [1], the import of modeling software airfoils in together as sample line to pay attention to don't together as a whole, rather than segmented connection as shown in figure 1, the entire front points being on the leading edge is divided into two segments, trailing edge chamfer is a circular arc, and the upper boundary and lower boundary line a total of five samples, this is in order to carries on the wing surface envelope into entities later lay a node, the framework of early but the leading edge of airfoils on is at the origin $(0,0)$, so will airfoils under translation makes the airfoil boundary point and the origin of overlap, before that, first of all, as a leading edge and trailing edge chamfer the connection to the center of the circle, the line is coincidence with strings of airfoil, this is for later at different radius airfoils blade Angle of torsion prepared a auxiliary line.

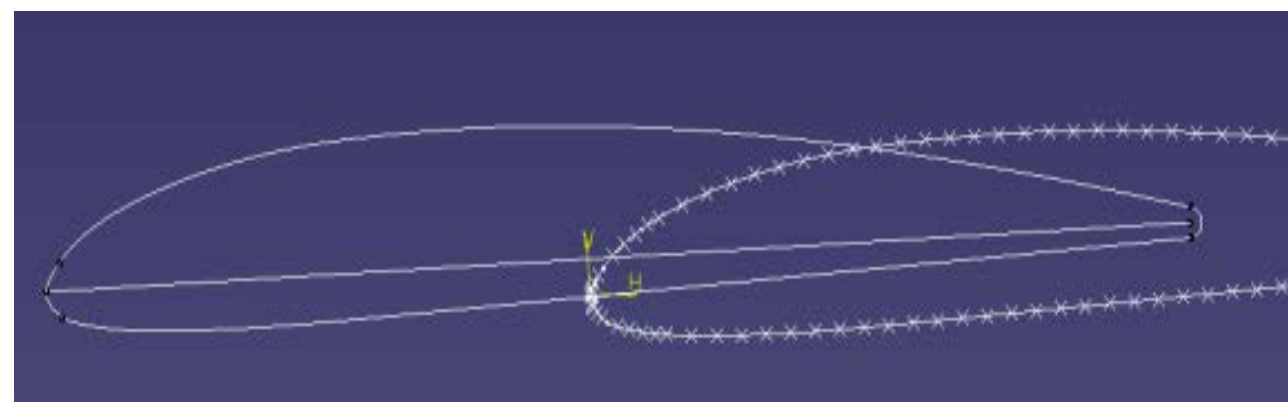

Fig. 1 Basic airfoil support preparation 


\section{Establishment of Frame Structure}

Next after the translation of the airfoil combined into a whole, along the center of the circle in the exhibition to be a auxiliary line length is $500 \mathrm{~mm}$, the line is also blade shaft centerline of variable pitch, and then along the line the airfoil translation copy of 10, the spacing between the two $50 \mathrm{~mm}$, new ten airfoil's position at the right moment is the propeller 10 benchmark stance, auxiliary strings from the baseline airfoil again also reproducing translation.

After the translation of the airfoil and airfoil string according to the section on $3 \mathrm{~d}$ geometric model data in the calculation of propeller blade Angle separately torsion, torsion Angle is at this time we do at first chord guides and horizontal plane Angle, airfoils with reverse string auxiliary line, as shown in figure 2.

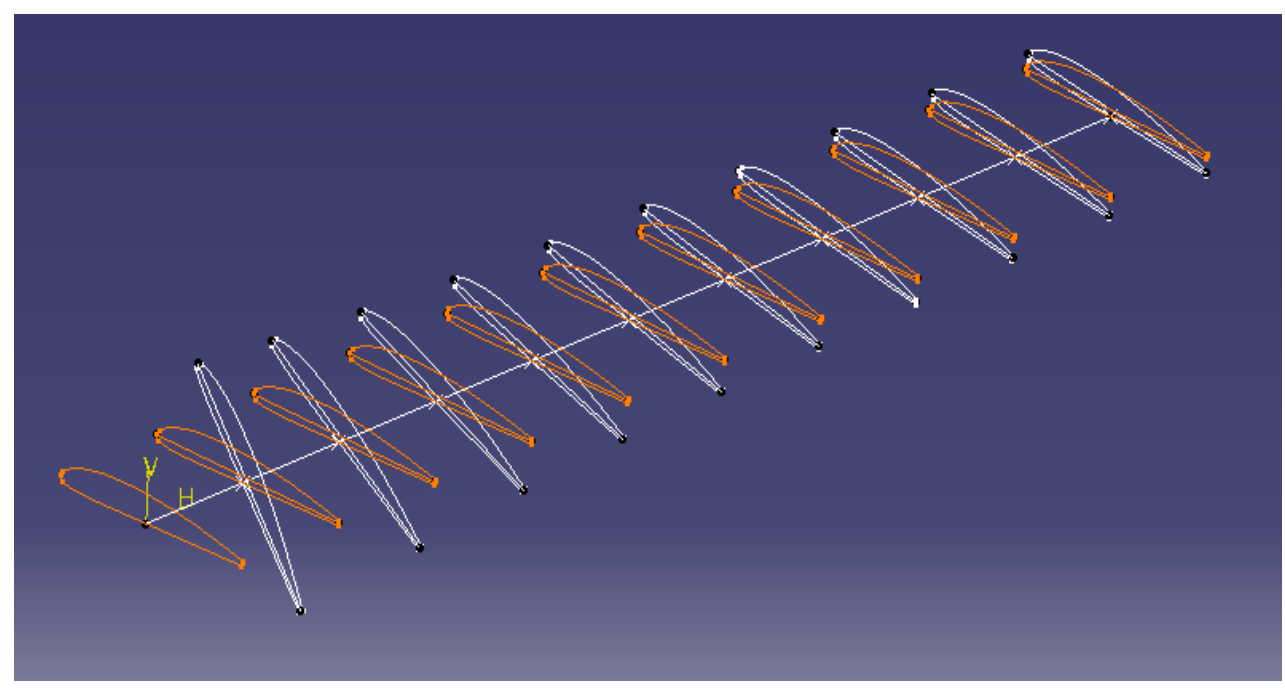

Fig. 2 The torsion of the airfoil

Airfoils, after the completion of reverse all the airfoil data X, Y, Z three directions to zoom in, the ratio of $\mathrm{X}$ to the ratio of 1 , referring to the blade show no scaling, and $\mathrm{Y}$, and $\mathrm{Z}$ to the refers to the ratio of the airfoil chord direction and thickness direction of the expansion ratio, [1] to get the specific ratio value is calculated individually for each direction of lofting, this way we can build the frame structure of the [2].

\section{Formation of Wing Surfaces}

When we are in front of the airfoil generation with the five sample line connection, the connection between the five lines of spline node is using the right now, we use airfoils on each of these nodes in the blade edge before and after can make five lines to wing surface samples, using multiple section again tools to wing surface envelope is shown in figure 3 .

\section{Establishment of the Benchmark Model}

Finally turn $180^{\circ}$ of the wing surface replication, and according to the geometric parameters of propeller hub, and the wing surface and cutting wheel nozzle-vessel junction place, finally to generate the benchmark model of propeller [3].

\section{Promotion of Basic Model}

There is the basis of the propeller model, then the size and structure of propeller model can be popularized in benchmark propeller model on the basis [4-5], such as P_4626 OARS, here in English letter "P" means the propeller "Propeller", the "46" here refers to 46 inches in diameter, "26" refers to the pitch of 26 inches, benchmark oar diameter of $1000 \mathrm{~mm}, \mathrm{P} \_4626$ oar diameter is $1168.4 \mathrm{~mm}$, so that you can get similar ratio 1.1684, the propeller of the other size according to the 
similar ratio of amplification, the change of particular attention to the pitch change makes the blade Angle, diameter change also cause the change of the blade Angle, because the new benchmark of propeller stance changed the spacing.

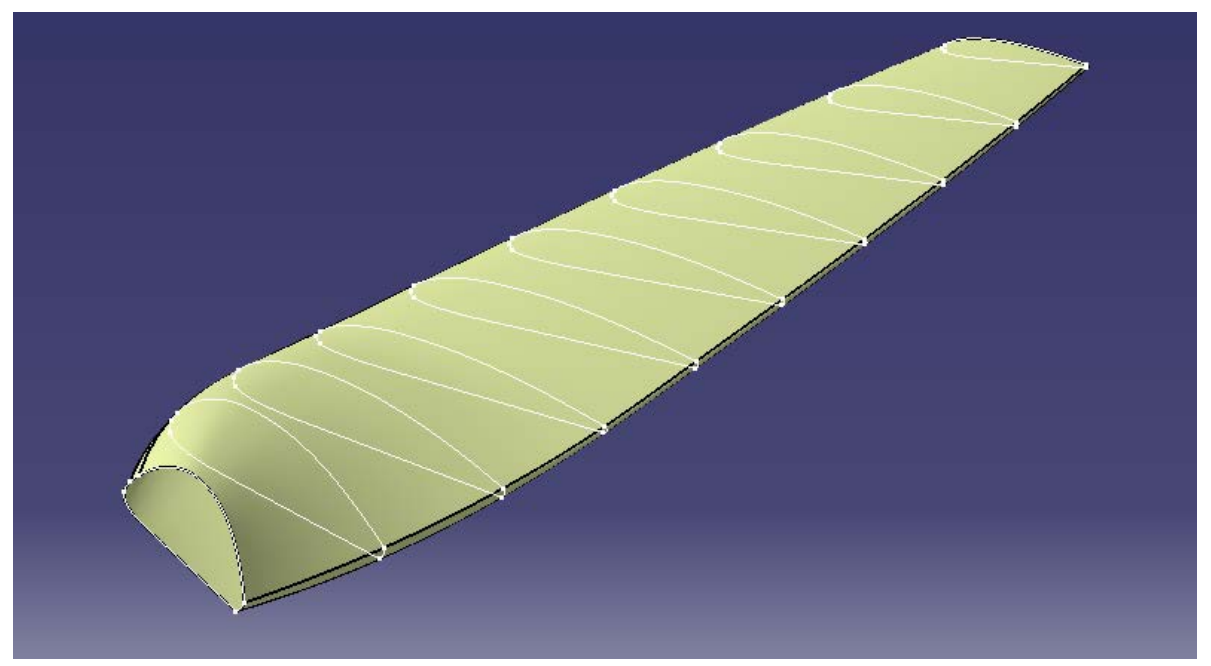

Fig. 3 Formation of airfoil

\section{Conclusion}

According to the above steps, and using relevant software we achieved by calculation propeller based model, in the process of building model involves many skills, there are a lot of innovative, and solves the questions about usually propeller model and difficulties, especially the established model can be the basis of promotion, it's easy to get what we need any propeller model of pitch, diameter size, brought great convenience to our study, for the UAV propeller performance study provides a certain basis and basis.

\section{References}

[1] Yang Yang, Cui Jinfeng, Yu Yi. Dynamic analysis and modeling of tri-rotor aircraft [J]. Optical Precision Engineering, 2013,07 (7):1873-1880.

[2] Wang Shi, et al. Helicopter aerodynamics [M]. Nanjing University of Aeronautics and Astronautics, 1976

[3] Formation Flight Problems [J]. Flight Mechanics, 2003,21(2):5-7

[4] Qi Jun, Xu Guohua. Design techniques for rotor meshing of blade movement. Journal of Nanjing University of Aeronautics and Astronautics, Vol. 36, No. 3, 2004:288-293

[5] Liu Lili. Modeling and control methods of the four-rotor flight simulator [D]. Central South University, 2009. 